
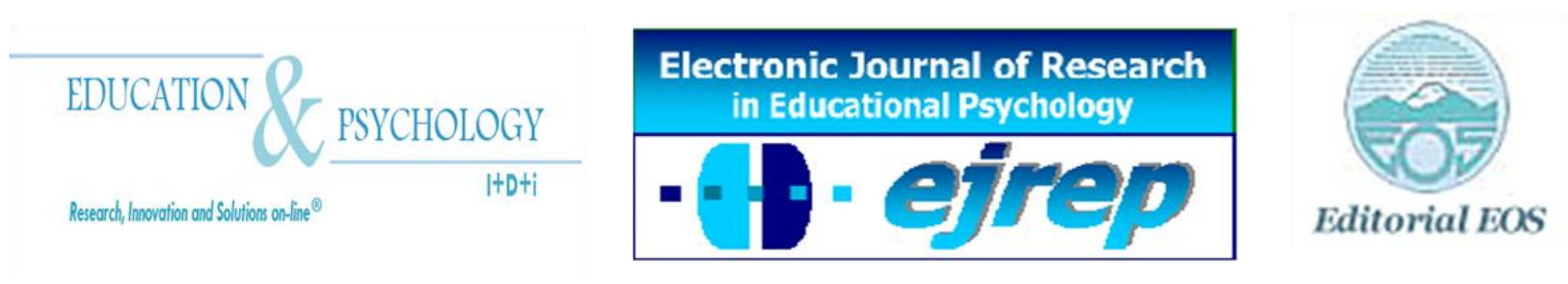

\title{
Procesos de Resolución de Problemas y Videojuegos: el Caso de Sim City Creator
}

\section{Natalia Monjelat ${ }^{1}$, Laura Méndez Zaballos ${ }^{2}$ y Pilar Lacasa ${ }^{3}$}

${ }^{1}$ Departamento de Filología, Documentación y Comunicación Audiovisual, Universidad de Alcalá, Alcalá de Henares

${ }^{2}$ Departamento de Psicología Evolutiva y de la Educación, Universidad Nacional de Educación a Distancia UNED, Madrid

${ }^{3}$ Departamento de Filología, Documentación y Comunicación Audiovisual, Universidad de Alcalá, Alcalá de Henares

\section{España}

Correspondencia: Natalia Monjelat. Calle San Cirilo s/n. Aulario de María Guzmán. 28801, Alcalá de Henares, Madrid, España. E-mail: natalia.monjelat@uah.es 


\section{Resumen}

Introducción. Los videojuegos han probado ser un recurso válido para trabajar diferentes cuestiones escolares. Más allá de contenidos puntuales, permiten trabajar distintas habilidades, dentro de las cuales podemos destacar la resolución de problemas. Sin embargo, no mucho se ha estudiado sobre esta temática o muchos estudios toman una perspectiva no del todo compatible con un enfoque educativo.

Método. En este trabajo analizamos los procesos de resolución de problemas que se presentan en un aula al introducir el videojuego comercial Sim City Creator tomando como referencia la teoría del procesamiento de la información. Los datos provienen de un estudio etnográfico llevado a cabo en un instituto secundario de Madrid, con alumnos de $3^{\circ} \mathrm{ESO}$, que utilizaron Sim City Creator en la asignatura de Ámbito Socio-Lingüístico, y han sido analizados desde un enfoque cualitativo y de estudios de casos considerando también una perspectiva del análisis del discurso.

Resultados. Cuando los estudiantes se enfrentan a los problemas que presenta el videojuego, construyen representaciones del mismo. Para ello identifican los operadores necesarios, localizados en el menú del juego, atendiendo a condiciones derivadas del docente, de los mismos estudiantes o del juego, que acotan el espacio del problema. Asimismo, para resolver los problemas los estudiantes recurren a diferentes estrategias, dentro de las cuales se destacan en nuestro estudio el uso de esquemas previos y el establecimiento de submetas.

Discusión y conclusión. Los videojuegos comerciales, utilizados en entornos educativos, pueden convertirse en recursos valiosos para trabajar los procesos de resolución de problemas. El apoyo del docente y su conocimiento del juego resultan un elemento valioso a la hora de sacar partido al videojuego, favoreciendo la construcción de las representaciones de los diferentes problemas.

Palabras Clave: Resolución de problemas, videojuegos, educación secundaria, nuevas tecnologías en educación. 


\begin{abstract}
Introduction. Video games have proven to be a valuable resource to work different school subjects and topics. Beyond specific content, they could help to develop different habilities, like problem solving. However, not much have been studied on this topic, or many of the studies followed a perspective not entirely conpatible with an educational approach.

Method. Based on the theory of information processing we analyze the problem-solving processes presented in a classroom when the commercial game Sim City Creator was introduced. The data come from an ethnographic study conducted in a secondary school in Madrid, with students of $3^{\circ}$ ESO, where Sim City Creator was used in Socio-Linguistic Area. Qualitative approach and case study perspective along with discourse analysis were the frame for the data analysis.
\end{abstract}

Results. When students are faced with the problems of the game, they build representations. To do so, they identify the necessary operators, located in the game menu, also considering the conditions exposed by them, the teacher or the game play, which narrow the problem space. Also, in order to solve the problems students use different strategies, among which we highlight the use of previous schemes and the establishment of subgoals.

Discussion and conclusion. Commercial video games used in educational settings can become valuable resources to develop problem-solving processes. The support of teachers and their knowledge of the game are a valuable asset when it comes to take advantage of the game, favoring the construction of representations of the different problems that appear during the game.

Keywords: Problem solving, video games, secondary school, new technologies in education

Received: 07/30/12 Initial acceptance: 08/07/12 Final acceptance: 11/08/12 


\section{Introducción}

Los videojuegos han demostrado ser un importante recurso educativo (Gee, 2003, 2008; Jenkins, 2003, 2006a, 2006b; Lacasa, 2006, 2011; Prensky, 2001, 2002; Salen \& Zimmerman, 2004; Squire, 2003) siendo utilizados dentro de la escuela con diferentes finalidades, como por ejemplo, la resolución de problemas, temática ampliamente abordada por el campo de la psicología (de Vega, 1998; Duncker, 1945; Holyoak \& Morrison, 2005; Robertson, 2001). Sin embargo, no mucho se ha trabajado en torno a los procesos puntuales que se llevan a cabo cuando se resuelven problemas presentados por los videojuegos. Las investigaciones recientes sobre resolución de problemas (Pizlo, 2009) revelan que en su mayoría se realizan en situaciones experimentales donde los mismos se analizan desde una perspectiva del desarrollo o desde la neurociencia, mostrando evidencia basada en estudios con imágenes, o presentando investigaciones relacionadas con las matemáticas, algebra o aritmética. Asimismo otros estudios presentan análisis más cuantitativos que no necesariamente se centran en el proceso de resolución de problemas en sí, sino más bien en los resultados de éste (Lee, Koh, Cai \& Quek, 2012).

Sin desmerecer esas propuestas, pensamos que el enfoque del procesamiento de la información (Bassok \& Novick, 2012; Newell \& Simon, 1972; Novick \& Bassok, 2005; Simon, 1978) puede ofrecernos un análisis diferente de los fenómenos a estudiar, permitiéndonos establecer conexiones con las características del propio videojuego y del contexto en el que se ha empleado (Lacasa, 2011). Basándonos en este enfoque teórico, nos interesa reflexionar sobre los procesos que se desarrollan cuando el alumnado resuelve los problemas que plantea el videojuego de simulación Sim City Creator. Nos interesa analizar el papel de los operadores y las condiciones para construir una representación adecuada del problema, presentando también algunas formas de solucionarlos utilizando esquemas previos o estableciendo submetas.

\section{Resolución de problemas y videojuegos: buscando puntos de enlace}

No es sencillo establecer relaciones entre estos dos campos que a simple vista parecen dispares sin comprender primero sus particularidades. Presentaremos la concepción de resolución de problemas adoptada en este trabajo, para luego señalar las relaciones entre dicha teoría y las características propias de los videojuegos utilizados dentro de un taller escolar. 


\section{El proceso de resolución de problemas}

Podemos decir que una persona se enfrenta a un problema cuando quiere algo y no sabe inmediatamente qué tipo de acciones debe realizar para lograrlo (Newell \& Simon, 1972). Resolver un problema implica una interacción entre un sistema de procesamiento de la información, el sujeto y el ambiente de la tarea (Simon, 1978). Cuando el sujeto resuelve un problema, enfoca la tarea y representa la situación en lo que se denomina espacio del problema, que es la forma en que la persona se representa el ambiente de la tarea enunciado por el investigador (Simon, 1978). Construir una representación adecuada y completa del problema es clave para alcanzar su solución (Domin \& Bodner, 2012; Jonassen, 2011, Robertson, 2001). Esta representación de la tarea a resolver incluye cuatro elementos (Bassok \& Novick, 2012; Newell \& Simon, 1972; Novick \& Bassok, 2005) señalados en la figura 1_que se incluye a continuación:

- El estado inicial: implica lo que se conoce sobre el problema antes de intentar resolverlo

- El estado meta: se refiere a lo que se quiere conseguir una vez resuelto el problema

- El conjunto de operadores: son las acciones posibles o los pasos a dar para poder alcanzar el estado meta

- Las condiciones: que indican lo que no es permitido y las consecuencias de escoger una u otra acción.

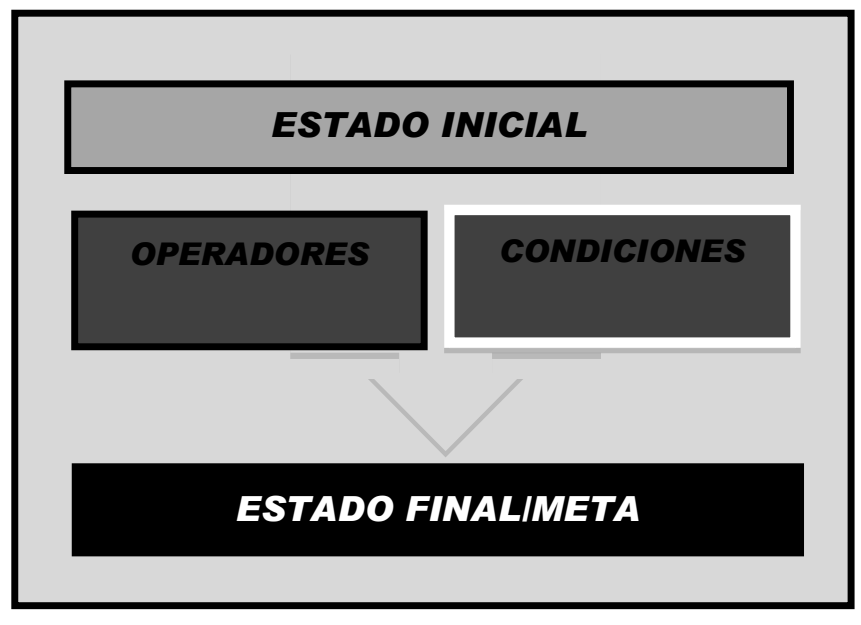

Figura 1. Elementos del espacio del problema

Por otra parte, la representación que la persona construye del problema puede ser interna o externa (Hayes, 1989; Novick \& Bassok, 2005). Las primeras implican objetos y relaciones mentales que se corresponden con los objetos y relaciones del problema presentado 
externamente. A veces una representación interna es suficiente pero en otros casos se necesita de una representación externa. Dibujar, escribir símbolos o demás ayudas pueden servir para establecer correspondencias con partes de la representación interna.

Según Hayes (1989) para formar una representación interna adecuada se debe "representar su meta y además, en los problemas que lo requieran, el estado inicial, los operadores y las restricciones de los operadores" (p. 7). De esta forma, se incluyen los componentes del espacio del problema que planteaban Newell y Simon (1972). Crear una representación interna implica una selección de información, estableciendo qué aspectos del problema son relevantes. Por ello no son una copia, ya que implican un proceso activo en el que la persona agrega, quita e interpreta información a partir de la situación original (Hayes, 1989). De esto se desprende la importancia que tiene el ofrecer al alumnado situaciones problemáticas que les permitan adquirir experticia en diferentes ámbitos. Resolver problemas usando videojuegos puede ser una manera de adquirir conocimiento y aumentar las experiencias previas, lo cual puede ser útil para enfrentarse a otras problemáticas.

\section{Estrategias de resolución: submetas y esquemas}

Una vez que hemos reconocido la existencia de un problema y creado su representación, hay que resolverlo. Para ello se pueden recurrir a diferentes estrategias entendidas como caminos que permiten alcanzar la solución (Lacasa, 2011). Algunas son más simples, como el ensayo y error, donde las personas no tienen o no utilizan información que les indique cuál es el camino que más factiblemente los lleve a la meta. Este método puede ser efectivo cuando el espacio del problema es pequeño (Hayes, 1989), pero en otros casos se precisan otras estrategias como por ejemplo los métodos de proximidad que utilizan diferentes heurísticos (Novick y Bassok, 2005). Uno de ellos es hill climbing (la escalada) en donde en cada paso, se aplica un operador que lleva a un nuevo estado que parece ser el más similar al estado-meta. Según Novick y Bassok (2005) otro método ampliamente utilizado es mean-ends analysis (análisis medios y fines), más sofisticado que el primero, ya que no depende de la similitud con la meta. La diferencia entre ambos heurísticos reside en la generación de submetas que permite el segundo, ya que las mismas aportan dirección, y esto hace que se reduzca la brecha entre el estado inicial y el final (Hayes, 1989). Las submetas han sido identificadas como componentes de la estructura de la tarea que pueden ser enseñados a los aprendices (Catrambone, 1996). Otra estrategia a destacar son los métodos fraccionarios que consisten en dividir el problema 
en sub-problemas. De esta forma, también se trabaja con submetas que la persona deberá identificar (Hayes, 1989).

Por otra parte, en algunas situaciones problemáticas las personas recurren a esquemas empleados previamente. Un esquema es un paquete de información sobre las propiedades de un tipo de problema en particular en donde sus elementos (estado inicial, meta, operadores y condiciones) se presentan de una determinada manera (Hayes, 1989). Los esquemas permiten a las personas reconocer experiencias similares y recuperar de la memoria el marco que se ha creado previamente para enfrentar la nueva situación problemática (Marshall, 1995). En este sentido podemos decir que al emplearlos se están utilizando métodos basados en el conocimiento (Hayes, 1989) ya que la persona recurre a lo que ha aprendido a través de los problemas que ha resuelto anteriormente, utilizando todos esos saberes para enfrentarse a la situación actual (Novick \& Bassok, 2005). También se ha comprobado que las imágenes presentadas en formato de vídeo ayudan a establecer relaciones y a recuperar esos esquemas (Goh, Tan \& Choy, 2012) por lo que los videojuegos pueden favorecer este tipo de transferencia. Asimismo, cuando se utilizan estas representaciones previas se está resolviendo el problema por analogía. Como señalan Gick y Holyoak (1980) “en esencia, la analogía y el esquema consisten en un sistema organizado de relaciones" (p. 309). Asimismo, Holyoak (2005; 2012) agrega que dos situaciones son análogas si comparten un patrón de relaciones entre sus elementos constitutivos aunque estos mismos difieran en ambas situaciones. Este tipo de razonamiento es común en la vida diaria y resulta útil para resolver situaciones en la vida real.

Aprender a resolver problemas no es tarea sencilla, saber elegir la estrategia adecuada tampoco. Sin embargo, ya que éstos se presentan día a día en las vidas de las personas, resulta indispensable buscar formas de enseñar a los jóvenes a desarrollar estrategias de resolución de problemas. Johnson-Laird (2008) señala que los individuos no vienen equipado con ellas, sino que las desarrollan sobre todo, al enfrentarse varias veces a situaciones similares. De esta manera podemos pensar que si ofrecemos a los estudiantes experiencias en donde se pongan en juego las mismas, será más posible que las adquieran. En este sentido, los videojuegos resultan instrumentos valiosos a la hora de pensar en escenarios educativos que busquen desarrollar los procesos de resolución de problemas, ya que ofrecen constantemente desafíos a resolver. 
Videojuegos y resolución de problemas: una propuesta de integración

Diversos autores mencionan que los videojuegos permiten trabajar procesos de resolución de problemas (Egenfeldt Nielsen, Smith \& Tosca, 2008; Gee, 2007, 2008; Juul, 2005; Squire, 2003) aunque como hemos señalado, no se profundice en el cómo llevarlo a cabo. Sin embargo, para estos autores resulta claro que este recurso permite resolver problemas y que tiene características particulares que resaltan su potencial para ello (Lacasa, 2011). Para entrar en tema, es necesario entonces señalar cuáles son las características generales de los videojuegos que nos permitirán construir una representación del mismo, yendo más allá del sentido común y la informalidad de la vida diaria (Egenfeldt-Nielsen et al., 2008). En nuestro trabajo consideramos que los videojuegos son sistemas basados en reglas (Juul, 2005) en donde se busca alcanzar un resultado a través del esfuerzo del jugador que controla el juego. Esta definición resalta el hecho de que los juegos son sistemas que tienen resultados precisos (Egenfeldt-Nielsen et al., 2008). En base a ella, Juul (2005) estable la diferencia entre juegos y nojuegos, señalando además casos limítrofes (bordeline), dentro de los cuales ofrece el ejemplo de Sim City, videojuego utilizado en nuestra investigación. Esto se debe al carácter libre de este juego, en donde no hay una meta bien definida que indique que se ha "ganado", pero sin embargo, sí que se pueden establecer diferentes metas y submetas que responden al objetivo del juego: crear, construir y gestionar una ciudad virtual (Monjelat, 2009).

Por otra parte es importante señalar que los videojuegos permiten "aprender haciendo" (Shaffer, 2004). De esta forma el aprendizaje es un proceso de constante práctica e interacción en tareas cada vez mas desafiantes, donde los jugadores gradualmente revelan sistemas de reglas subyacentes (Sandford \& Williamson, 2005), que son importantes a la hora de resolver los problemas que plantea el videojuego. En este sentido el diseño del juego puede plantear ciertas reglas que funcionan como condicionantes estableciendo lo que está permitido y lo que no durante la partida. Otro punto a tener en cuenta es la posibilidad que ofrece el videojuego de entrar en mundos virtuales (Shaffer, 2006) donde se pueden entender conceptos complejos sin perder las conexiones entre las ideas abstractas y los problemas reales. Los videojuegos de simulación, debido a las características señaladas motivan a los aprendices a explorar y jugar (Baek, 2010) incluyendo principios educativos (Gee, 2007) que hacen que sean un poderoso recurso educativo. 
En nuestro trabajo analizaremos como se ha utilizado el videojuego Sim City Creator para resolver problemas en un taller realizado con alumnos y alumnas de $3^{\circ} \mathrm{ESO}$, que será descripto en profundidad en posteriores apartados. Teniendo en cuenta las características señaladas de los videojuegos y los procesos de resolución de problemas expuestos previamente, hemos desarrollado una figura donde se reseñan las relaciones entre dicho modelo teórico, las características del videojuego y del contexto donde se ha empleado. La figura 2 que se presenta a continuación es el punto de partida de nuestro trabajo y resume estas cuestiones.

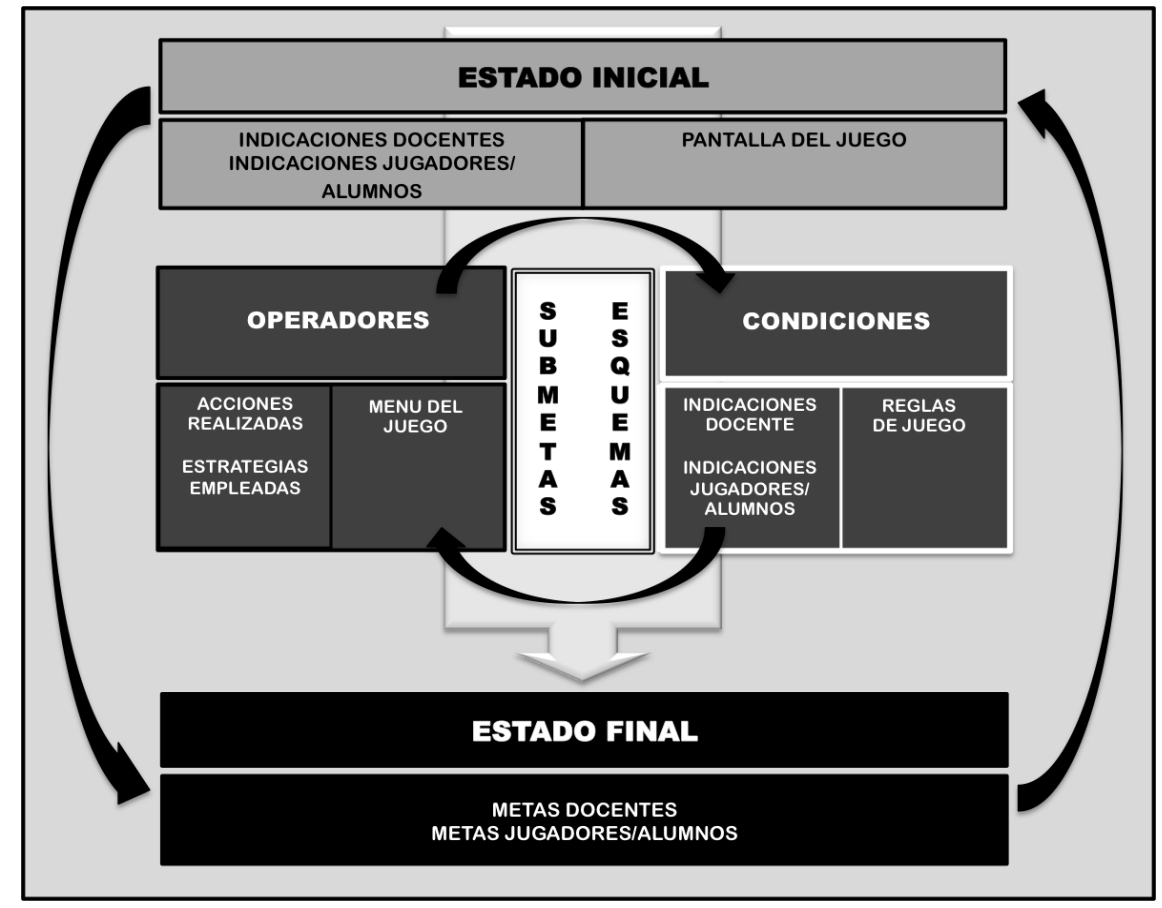

Figura 2. Representación del problema en función de las características del taller

En ésta podemos observar como los diferentes elementos del espacio del problema incluidos en la representación del mismo, se materializan en función del contexto propio de la investigación, cuyos componentes principales son: los participantes, el videojuego y la resolución de problemas. Analizando la figura se observa como el estado inicial se corresponde en líneas generales con las indicaciones del docente o de los mismos alumnos, pero al estar jugando, es necesario también tener en cuenta la información que nos ofrece la pantalla del juego, como punto de partida. El estado final, la meta de la partida, puede estar planteada también por el docente o por los propios alumnos debido a qué, como señalábamos, el juego es abierto y no plantea objetivos puntuales.

Para cruzar la brecha desde el estado inicial al final, necesitamos encontrar los operadores, que en el caso del videojuego hacen referencia a los iconos del menú del mismo, que 
permiten llevar a cabo las acciones y estrategias necesarias para avanzar. Para elegir adecuadamente los operadores, es necesario considerar las condiciones que establezcan lo que puede hacerse, y lo que no. En este caso, las mismas suelen provenir del docente o los alumnos, pero también pueden relacionarse con reglas propias del juego o con su diseño. Asimismo hemos agregado los conceptos de submeta y de esquema, para referirnos a las etapas intermedias en el proceso, que derivan de la interacción entre las condiciones y los operadores siendo estrategias de resolución del problema. De esta forma, esta figura nos permite revisar cómo se plantea un modelo clásico de resolución de problemas en un ámbito particular, en este caso, cuando se usan videojuegos en la escuela.

\section{Estudiando los procesos se resolución de problemas: marco metodológico}

La investigación que presentamos se desarrolla siguiendo un enfoque etnográfico (Anderson-Levitt, 2006; Lacasa \& Reina, 2004) y realizando un análisis cualitativo (Denzin \& Lincoln, 2011) desde un estudio de casos (Stake, 2006; Yin, 2011) incluyendo también una perspectiva del análisis del discurso (Gee, 2011; Gee \& Green, 1998). Este tipo de metodología ha recibido diferentes críticas en torno a su validez cuando se la compara con estudios cuantitativos que emplean métodos estadísticos o buscan generalizaciones (Anfara, Broen \& Mangione, 2002; Flyvbjerg, 2006; Hoepfl, 1997; LeCompte \& Goetz, 1982). Sin embargo, lo que puede ser interpretado como una limitación por otros autores, es lo que en nuestro caso justifica el uso de este enfoque. En ese sentido la etnografía, al enfatizar el interjuego de las variables en un contexto natural, permite un análisis profundo de las situaciones, lo que puede no estar presente en otros enfoques metodológicos (LeCompte \& Goetz, 1982). En nuestro estudio resulta apropiada, ya que desde la misma, la investigación no basa su validez en la frecuencia de aparición de cierto fenómeno, sino en la descripción detallada de casos (Ragin \& Becker, 1992; Stake, 1995) en los que se pueda explicar cómo las personas atribuyen sentido a su actividad en contextos socioculturales definidos (Lacasa, 2011; Lacasa, Méndez \& Martínez, 2008; Spindler \& Hammond, 2000). En ese sentido nos interesa analizar las representaciones compartidas de los problemas que se han generado en una situación escolar. En este contexto, nuestro objetivo consiste en analizar los procesos involucrados en la resolución de problemas dentro del contexto del aula, donde se trabajó con el videojuego comercial Sim City Creator para la consola Wii. Presentaremos diferentes secuencias que se han desarrollado en el trascurso del taller y que dan cuenta del proceso de pensamiento que ha llevado a los participantes a cruzar la brecha entre el estado inicial y el estado final cuando se navega en el 
espacio del problema (Hayes, 1989, Simon \& Newell, 1972). Mientras jugaron los alumnos se enfrentaron a diversos problemas a la hora de construir los servicios e infraestructuras necesarios en la ciudad. En esa línea mostraremos las representaciones de esos problemas, analizando cómo se presentan sus elementos y cómo se han solucionado, retomando el marco teórico presentado previamente.

\section{Contexto y participantes}

Nuestro estudio se enmarca dentro de dos proyectos llevados a cabo por el grupo "Imágenes, Palabras e Ideas" de la Universidad de Alcalá. Uno en colaboración con la empresa Electronics Arts, como parte de su programa de responsabilidad social y el segundo, financiado por el Ministerio español de Educación y Ciencia dentro del plan nacional de I+D+I ${ }^{1}$. El mismo se desarrolló durante el curso escolar 2008-2009 (Lacasa \& GIPI, 2009) en un instituto de educación secundaria, al sur de la Comunidad Autónoma de Madrid. Los datos presentados provienen de un taller llevado a cabo en la asignatura Ámbito socio-linguiístico de Diversificación Curricular, en el que participaron 10 alumnos de $3^{\circ}$ ESO y su profesor, trabajando con el videojuego comercial Sim City Creator. Este juego de simulación permite construir de una ciudad y gestionarla, asumiendo el rol de alcalde. En los talleres los alumnos jugaron con la consola Wii de Nintendo organizados en pequeños grupos de 2 o 3 estudiantes. En el análisis nos centraremos en uno de los grupos participantes del taller compuesto por María y Pedro².

\section{Diseño y desarrollo de la experiencia}

En el marco del proyecto de investigación se realizaron diferentes aproximaciones al escenario educativo antes de comenzar los talleres y la recogida de datos, que permitieron el acceso al ámbito de estudio (Lacasa et al., 2009; Lacasa, 2011). Como podemos observar a continuación en la figura 3 se realizaron diferentes actividades iniciales que permitieron informar a los participantes sobre las cuestiones básicas del proyecto, atendiendo a sus consultas y permitiéndoles una primera aproximación al contexto del trabajo.

\footnotetext{
1 Proyecto "Aprendiendo con los Videojuegos" financiado por la empresa Electronics Arts; Proyecto "Nuevas Alfabetizaciones" financiado por Ayudas para la Realización de Proyectos de Investigación del Programa Nacional de Ciencias Sociales, Económicas y Jurídicas. Programa SEJ (Educación). Ministerio de Educación y Ciencia. Plan Nacional de I+D+i EDU2009-07075 (subprograma EDUC) (2008-2011). Natalia Monjelat cuenta con una beca del programa de Formación del Profesorado Universitario otorgada por el Ministerio español de Educación, Cultura y Deporte.

${ }^{2}$ Los nombres de los alumnos han sido modificados para preservar sus identidades
} 


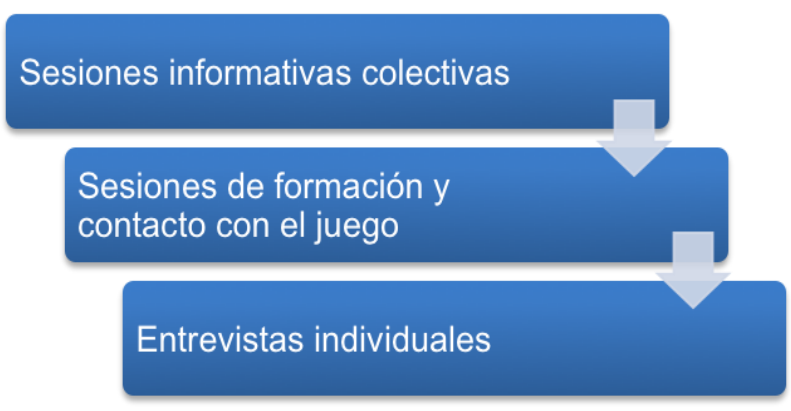

\section{Figura 3. Acceso al ámbito de investigación}

En base a las mismas y a los intercambios generados entre docente e investigadores, en el taller a analizar se estableció una planificación inicial de 7 sesiones semanales de 1 hora cada una, en las que los alumnos trabajarían por parejas o tríadas con el videojuego Sim City Creator, contando para ello con una consola Wii, una pantalla y 2 mandos inalámbricos. La figura 4 muestra la secuencia temporal del taller en un doble nivel, por una parte el conjunto de las sesiones y, por otra, qué ocurre habitualmente a lo largo de una determinada sesión. En ella podemos observar las diferentes fases del taller: la primera (4 sesiones) más centrada en el juego, y la segunda destinada a la realización de producciones audiovisuales (3 sesiones) que dieran cuenta de lo aprendido.

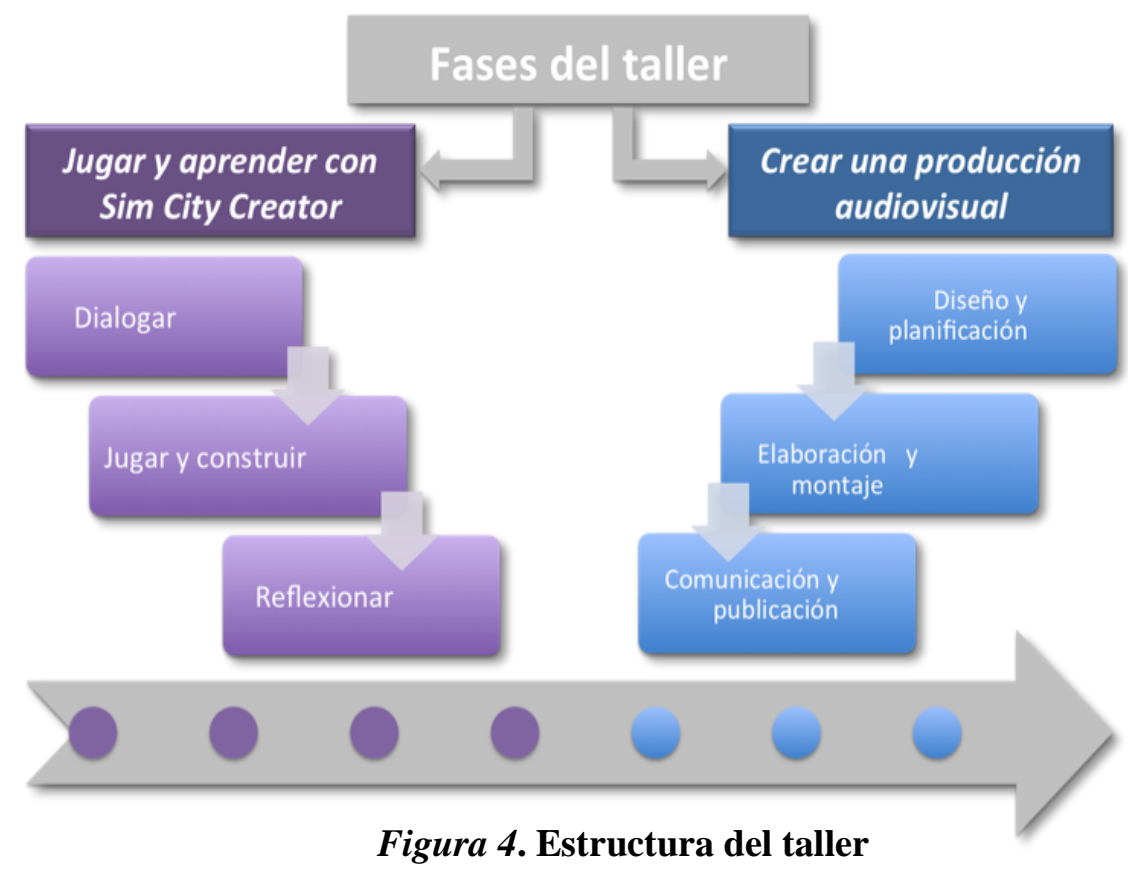

En el presente artículo nos centraremos en la primera fase, analizando fragmentos de la segunda sesión, donde observamos las interacciones que tuvieron lugar entre los participan- 
tes durante los momentos de juego, así como también las reflexiones y diálogos previos y posteriores en cada sesión.

\section{Toma de datos y análisis}

Cabe destacar que a lo largo del proyecto hemos tomado la posición de observadores participantes, recogiendo a su vez diferentes materiales que fueron de utilidad para el posterior análisis del taller. Como vemos en la figura 5, contamos con diferentes tipos de datos, en su mayoría audiovisuales (Pink, 2007).

\begin{tabular}{|c|l|l|l|l|}
\hline Curso & Sesiones & Fotografías & Grabaciones de audio & $\begin{array}{c}\text { Grabaciones en video } \\
\text { (Cámara fija y cámara móvil) }\end{array}$ \\
\hline $\begin{array}{c}\mathbf{3}^{\circ} \text { ESO } \\
\text { Diversificación. } \\
\text { Ámbito } \\
\text { Socio Linguistico }\end{array}$ & 7 & 208 & $2: 05: 43$ & $7: 24: 11$ \\
\hline
\end{tabular}

\section{Figura 5. Material recogido}

Los datos recogidos se analizaron a través del programa informático Transana que facilitó una primera aproximación a los mismos, permitiendo manejar con gran facilidad las grabaciones digitalizadas de audio y video, seleccionando los fragmentos más reveladores en función de nuestros intereses. Organizados y visualizados los datos, se realizaron sus transcripciones, que permitieron contar por escrito con las secuencias de dialogos que tuvieron lugar a lo largo del taller. En base a éstas se pudieron establecer niveles de análisis que permitieron reflejar los procesos de resolución de problemas que nos interesa abordar.

\section{Resultados y discusión}

Centraremos nuestro análisis en dos cuestiones claves para resolver un problema. Por un lado señalaremos la importancia de los operadores y las condiciones para construir una representación adecuada, señalando cómo los mismos se presentan en los problemas que plantea el videojuego. Por otra parte nos centraremos en el papel de las submetas y los esquemas para señalar diferentes formar de acercarnos a la solución de aquellos problemas que requieren una estrategia de resolución más compleja. 
Aproximándonos al problema: operadores y condiciones

Al enfrentar un problema se construye una representación interna del mismo que orienta los pasos para alcanzar la meta (Bassok \& Novick, 2012; Hayes, 1989). En este apartado analizaremos cómo los participantes han construido esas primeras representaciones y qué aspectos relacionados con el propio taller y con el videojuego han influido en ese proceso.

Localizando los operadores en el menú del juego: condiciones del juego y de los participantes

El menú del juego es amplio y los jugadores deberán escoger los iconos que servirán de operadores permitiéndoles resolver los diferentes problemas del juego. En la figura 6 observamos los tres niveles que el menú ofrece. Los mismos están agrupados por categorías, de manera que dentro de "Construcción" encontramos por ejemplo "Transporte" o "Servicio Público", y dentro de este último, las opciones de construcción que pueden elegirse (por ejemplo en transporte: carretera recta, carretera curva, autopista, etc.).

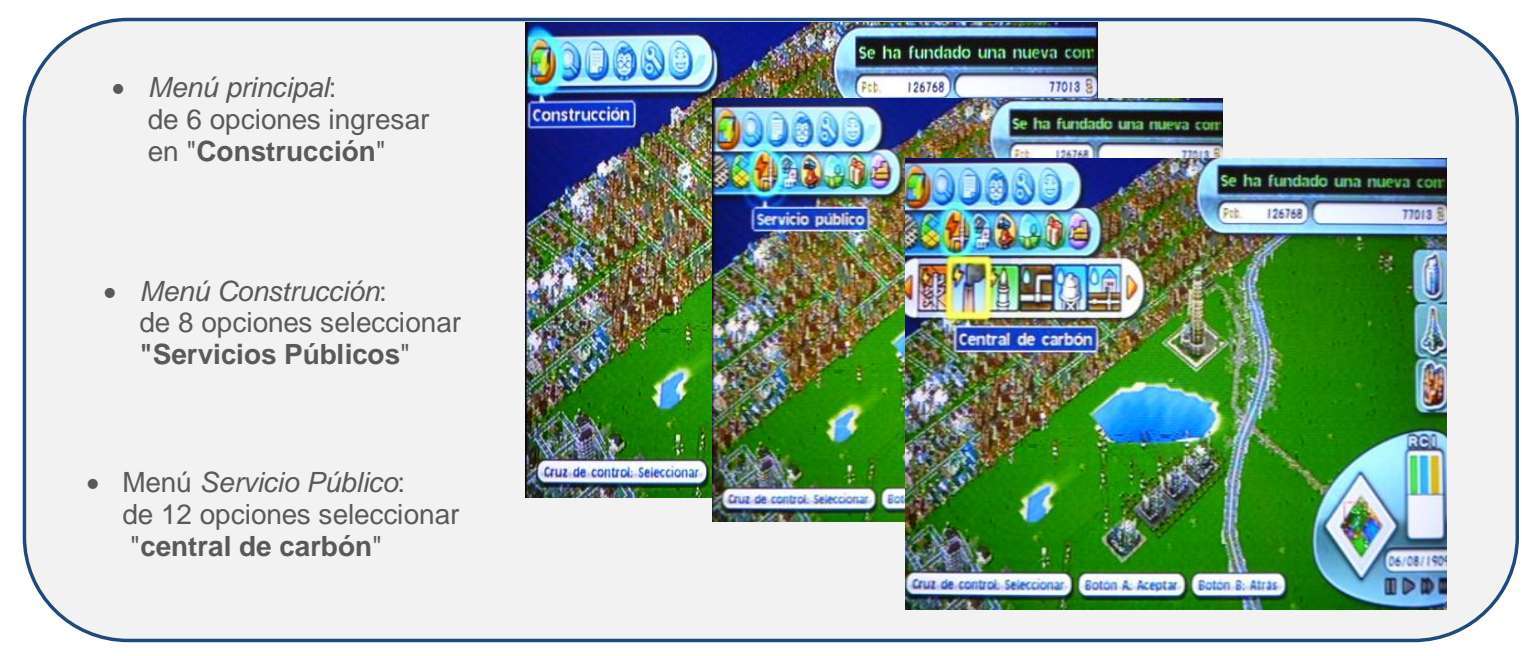

Figura 6. Niveles dentro del menú del juego. Un ejemplo

Debido a la complejidad del menú, encontrar los operadores no es sencillo, como veremos en los siguientes turnos donde alumnos y profesor resuelven el problema de suministrar electricidad a la ciudad. 
Fragmento 1: Localizando los operadores en el juego

200902 23. Sesión 2. Cámara móvil

1. Pedro ${ }^{3}$ : Dónde está el...

2. Prof.: El tendido, primero tienes que crear una central.

3. P: Pero es que idónde está?

4. Prof.: Las dos que tienes, las tenías ahí a las dos ((mirando la pantalla)). A la derecha tienes una ((se ve el icono para la central de carbón))

5. P: Y ésta ¿no?

6. P: ((La selecciona)) Pues ¿Dónde la ponemos?

7. María: por el medio no, porque ahí va a estar ((no se entiende))

Observamos como Pedro no está seguro de lo que debe hacer. Por ello, consulta al profesor sobre la ubicación de los elementos en el menú del juego como podemos ver en los turnos 3 y 5 (Pero es que ¿dónde está?; Y ésta ¿no?). Aunque tiene claro la meta, aún no identifica adecuadamente los operadores. Es por ello que el docente acota el espacio del problema, señalando condiciones (Bassok \& Novick, 2012; Newell \& Simon, 1972) que permiten a los alumnos localizarlos. Por una parte, se observa como en el turno 2 (El tendido, primero tienes que crear una central) el docente determina una secuencia de pasos (Novick \& Bassok, 2005), indicando condiciones a la hora de construir los elementos. Las reglas del juego (Juul, 2005) establecen que para construir la electricidad necesitamos del tendido y la central, sino contamos con ambas, no se considera que la ciudad tenga instalado el servicio. Sin embargo, el juego en su diseño no establece un orden para ello y no importa cuál se construye primero, siendo el docente quien establece ese orden.

Por otra parte, en el turno 4 (Las dos que tienes, las tenias ahí a las dos) el docente vuelve a ayudar a los alumnos a encontrar los operadores ubicándolos en el menú. Una vez identificado el operador, el icono de la central eléctrica de carbón, los estudiantes se preguntan en qué parte de la ciudad colocarla. En ese sentido, también observamos ciertas condiciones, pero en este caso, por parte de María que señala donde no piensa que debe colocarse la central en el turno 7 (por el medio no...). El juego permite hacerlo donde se desee pero sin embargo hay cuestiones que esta alumna tiene en cuenta, aunque desgraciadamente el audio no permita terminar de comprender su frase. En esa línea vemos como las reglas del juego sólo estipulan que no puede colocarse sobre otro objeto o sobre el agua, pero allí también en-

\footnotetext{
${ }^{3}$ En las transcripciones (P) corresponde a Pedro, (Prof.) al docente y (M) a María.
} 
tra en juego el criterio del jugador, que aprovecha esos parámetros flexibles para ubicarla donde mejor le parece.

Aunque se ha avanzado hacia el estado meta, aún nos falta colocar la otra parte necesaria para poder contar con la instalación completa del servicio: el tendido eléctrico. Una vez colocada la central, los alumnos se dedican a su construcción.

8. P: Ay que ya perdí la flecha ((se para y luego se sienta)) ¿Qué hacemos?

9. P: ((Selecciona del menú y coloca el tendido))

10. M: ...Pero de eso hay que hacer más io no?

11. P: Pues mira, ¿sabes lo que vamos a hacer?

12. M: Una $\mathrm{c}^{* * * * *}(($ se ríe $))$

13. P: Ehh, Ahí ((lo ha colocado en un extremo de la ciudad)), y así ya lo rellenamos todo, lo hacemos como un cuadradito así.

En los diálogos se observa como al haber recorrido el espacio del problema (Newell \& Simon, 1972; Simon, 1978) previamente, Pedro ya no tiene dificultades para localizar el icono del tendido que funcionará como operador. Una vez localizado, los alumnos tuvieron que pensar nuevamente en cuál era la mejor ubicación de estos elementos, reflexionando también sobre la estrategia para hacerlo. En este caso María señala una posible condición en el turno 10 (Pero de eso hay que hacer más ¿o no?) mientras que Pedro sugiere en el turno 13 colocarlo en forma de cuadrado para cubrir una mayor superficie. En este ejemplo podemos observar cómo es necesario cuando se juega, conocer los iconos presentes en el menú del videojuego que nos permitirán avanzar en el mismo. En este sentido las condiciones abren la posibilidad de localizar al operador, conocer la secuencia de pasos a seguir y decidir sobre la ubicación del elemento a construir.

En función de lo dicho, resulta interesante destacar que aunque el diseño del juego y sus metas sean abiertas (Juul, 2005), esto no impide que los participantes tengan sus propias metas y apliquen sus condiciones, que sumadas a las que se desprenden de las reglas del juego, permiten localizar los operadores y construir una representación del problema que procure resolver el mismo alcanzando el estado final. 
Elementos del juego y representación del problema

A veces construir una representación interna (Hayes, 1989) no resulta sencillo y es necesario contar con algunas ayudas extras. El problema del suministro de agua a la ciudad permite analizar estas cuestiones.

Fragmento 2: Elementos del juego que inciden en la representación del problema 200902 23. Sesión 2. Cámara móvil

1. M: ¿Y ahora qué?

2. P: Pues ahora hay que poner el agua.

3. M: ¿Adónde le doy? Toma ((le quiere dar el mando a Pedro))

4. P: ((no lo coge)) Dale a la "B", a la que está abajo ((le enseña dónde))

5. M: ¿Aquí? ((vuelve al menú))

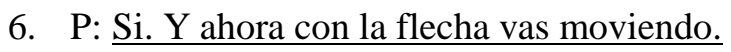

7. M: He perdido la flecha...

8. P: Con la flecha del mando.

9. M: Ahh $\downarrow$ No va Pedro...

10. P: Dale a la "A". Abajo. Ves ya te sale...

11. M: Ahh $\uparrow$

12. M: ¿A cuál le doy? ¿Crear zona?

13. P: Ahí, dale al "Servicio público" y ponemos el agua ya.

Observamos en este fragmento como pese a saber donde están los operadores en su conjunto, la alumna tiene dificultades con el mando inalámbrico de la consola que le impiden localizarlos con facilidad. Si no se controla adecuadamente el mando, se retrocede de nivel en el menú o se pierde de vista la flecha que permite ubicarse dentro de la pantalla del juego. De esa forma, resulta aún más difícil localizar el operador necesario, como podemos observar en la secuencia de turnos 5-11. En este caso, un elemento del propio juego no ayuda a construir la representación y a encontrar el operador sino que por el contrario, distrae la atención de la alumna hacia el control del mando, como se observa en una de las imágenes presentadas en la figura 7 , donde en vez de mirar la pantalla, la alumna se encuentra enfocada en el mando. 

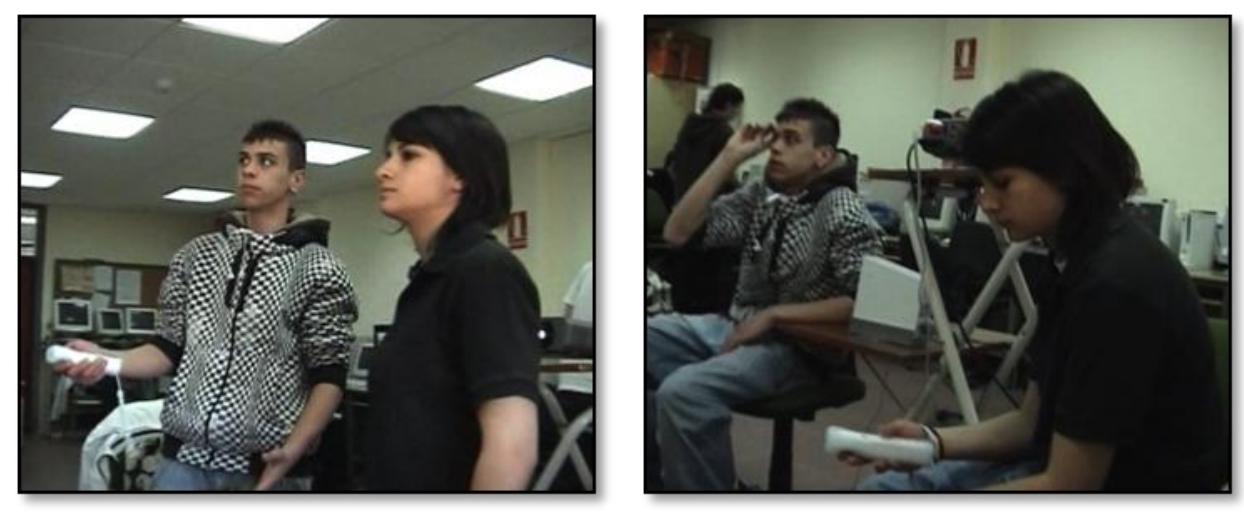

Figura 7. Alumnos interactuando con el mando inalámbrico

Por otra parte, como observamos en el turno 12 (¿A cuál le doy? ¿Crear zona?) la alumna desconoce la función que tienen los iconos del menú, ya que para colocar el agua, pretende utilizar el icono de crear zona. Cada icono del menú genera una acción diferente que permite avanzar hacia la meta o por el contrario, elegir un camino equivocado. En ese sentido el menú del juego puede ser una ayuda para encontrar los operadores y de esa manera se constituye en una representación externa (Zhang, 1999). Por otra parte, en los siguientes turnos observamos otro elemento que también cumple esa función.

14. M: ((la selecciona)) Y madre mía, ¿dónde lo meto a esto?

15. Prof.: Cerca de la población

16. M: Todavía no hemos creado la población...

17. Prof.: ¿Dónde esta la población? ¿No estaba al este?

18. P: $\mathrm{Si}$, arriba a la derecha.

19. Prof.: Al este, vamos a manejarnos con el mapa. Por ahí estaba la población ¿no? Eso es el este, sería más bien en el noreste. Pues ahora ahí en el noreste constrúyelo, no no, un poquito más...

En estos diálogos se hace evidente la función del mapa en el proceso de resolución del problema, como otro elemento, en este caso del propio juego, que ha ayudado a los alumnos a construir la representación del problema. En el videojuego la ciudad virtual esta construida sobre un mapa que puede observarse desde diferentes ángulos. De esta forma, el mapa funciona como una ayuda del propio juego para comprender el problema, representarlo y pensar su solución. Los alumnos se han valido de las palabras del docente, de sus condiciones y de los elementos del propio juego para colocar correctamente las arcas de agua y resolver esta parte del problema. La representación se ha ampliado (Hayes, 1989) incluyendo esta información y permitiéndoles avanzar hacia la solución 


\section{Submetas y esquemas: dos maneras de abordar problemas complejos}

Analizaremos dos formas diferentes de enfrentar algunos problemas del juego centrándonos en el papel que adoptan en el proceso las submetas y los esquemas, lo que supone una aproximación diferente frente a los problemas más complejos.

Estableciendo submetas para alcanzar la solución

En el apartado anterior observamos cómo los dos problemas expuestos (suministrar agua y electricidad) implicaban la construcción de ciertas infraestructuras para poder alcanzar el estado final. Resolver el problema implica entonces alcanzar ambos estados intermedios que en el caso de Sim City Creator, se materializan en la construcción de determinados elementos que serán seleccionados del menú del juego. Esta estructura organizada en submetas también está presente a lo largo del juego en otras secuencias, como por ejemplo, en la que nos muestra el fragmento 3 que analizaremos para profundizar en estas cuestiones.

Fragmento 3. Submetas para la construcción del ferrocarril 200902 23. Sesión 2. Cámara móvil

1. M: Qué hago...Madre mía, es que esto se mueve pa' todos los lados. Transporte ((mira las opciones del menú: transporte, salud, prevención de accidentes...))

2. P: Haz un tren o algo.

3. M: Bueno, esto va a tener de todo ¿y cuál es? ((busca en el menú)) Estación de tren ¿verdad? ¿Dónde la hago? ¿aquí mismo?

4. P: Donde tú quieras.

5. M: ((selecciona la estación de tren y la coloca en el mapa)) ¡Ay! ((por error vuelve al menú anterior y vuelve a ver las opciones)) Línea de metro, ah, o estación de metro ¿Qué hago Pedro?

6. P: Haz el ferrocarril ¿no? El tren

7. M: ((elige "ferrocarril" y comienza a construir las vías del tren realizando una especie de curva por toda la ciudad)) Madre mía, ya verás la que voy a liar. Voy a poner aquí...

8. P: Tú haz una curva todo grande, mientras que ocupe todo...

En el dialogo observamos como los estudiantes plantean en el turno 2 el estado final: la construcción de un tren ( $\mathrm{Haz}$ un tren o algo). Para alcanzar ese estado final es necesario nuevamente construir dos elementos, en este caso: la estación de tren y las vías del ferrocarril. En los diálogos correspondientes a los turnos 5-7 (-selecciona la estación de tren y la coloca en el mapa-, -elige "ferrocarril" y comienza a construir las vías del tren- ) observamos como 
los alumnos seleccionan del menú los elementos necesarios para luego ubicarlos en el mapa. De esta forma observamos en la figura 8 como se repite la estructura al igual que en los otros problemas donde para resolverlos ha sido necesario alcanzar dos submetas.

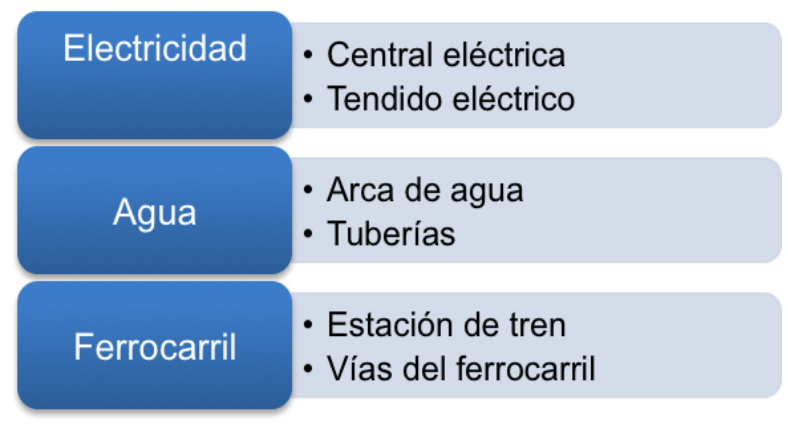

Figura 8. Submetas en cada problema

Este modo de resolver el problema responde a una método de fraccionamiento (Hayes, 1989), que divide el problema en submetas reduciendo la búsqueda dentro del espacio del problema. El diseño del juego es coherente en estos caso y para dar el servicio como construido requiere no sólo de ambos elementos sino también que los mismos estén conectados entre sí, lo cual es lógico, ya que en las ciudades reales funciona de esa manera. Es por ello que el tendido debe estar conectado a la central, las tuberías al arca, y las vías a la estación.

Esquemas previos para resolver problemas complejos

Al avanzar en la sesión observamos cómo los alumnos se enfrentaron a diferentes problemas. Han colocado agua y luz a la ciudad, construyendo a su vez carreteras y trenes. Estas experiencias les permiten adquirir conocimientos sobre la mecánica del juego y sobre sus reglas, que les serán útiles para resolver los problemas futuros (Novick \& Bassok, 2005). En algunos de los fragmentos destacados en los apartados anteriores se observa que al momento de realizar las acciones tendientes a resolver ciertos problemas, los estudiantes recurrieron a una misma estrategia a través de la cual, al construir, se pretende cubrir una gran superficie empleando el menor tiempo y esfuerzo posibles. Nos interesa entonces presentar un ejemplo más de esta forma de resolver el problema, que nos permitirá reflexionar sobre la utilización de los esquemas (Hayes, 1989) como estrategia de resolución. 
Fragmento 4: Utilizando esquemas previos

200902 23. Sesión 2. Cámara móvil

1. M: ¿A cuál le doy?

2. P: Ahí dale.

3. M: ¿Transporte?

4. P: Si ((miran las opciones del menú)) ¿Hacemos una autopista? Haciendo un circulo y ya... ((ambos se ríen) (...)

5. M: Una entrada a la autopista ¿no? Ahí, y ahora hacemos una carretera, o algo ahí ((señala con el mando el extremo del mapa))

6. P: Claro, unas curvitas... para que vayan por todos los lados.

En este ejemplo los alumnos construyen una autopista. En el turno 4 (Haciendo un circulo y ya), vemos como desean que sea circular. Asimismo, esperan colocar carreteras que también seguirán el mismo diseño, lo que se muestra el turno 6 (unas curvitas... para que vayan por todos los lados). Para Pedro, las curvas, los círculos y los cuadrados permiten abarcar grandes espacios con facilidad y es por eso que recurre a esa estrategia para realizar el tendido eléctrico, colocar las vías y en este ultimo ejemplo, las autopistas y carreteras. Dichas construcciones quedan reflejadas en la figura 9, que muestra capturas de los momentos del juego a los que estamos haciendo referencia.

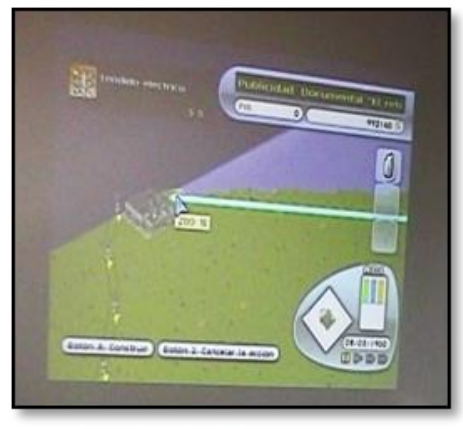

Cuadrado para el tendido, curvas para las carreteras y círculo para el ferrocarril
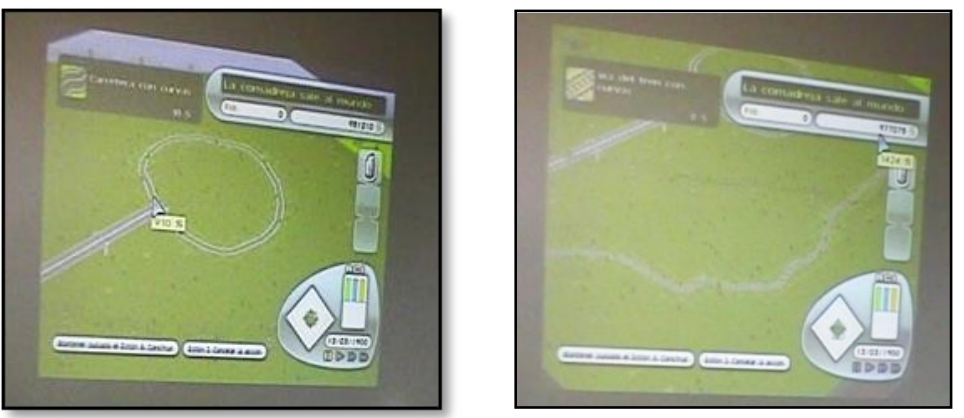

Figura 9. Esquemas

Como ya ha empleado esa estrategia, el alumno recupera el esquema que previamente ha generado para resolver un problema similar y lo emplea para llevar a cabo la nueva construcción (Marshall, 1995). Aunque cambie el servicio que se pretende suministrar (electricidad, carreteras o vías) la construcción de los mismos es análoga. Asimismo, lo dicho implica que se ha empleado el razonamiento por analogía (Gick \& Holyoak, 1980) al recuperar patrones similares e incluirlos en la representación del problema. En este caso al enfrentarse a la construcción del agua y el tren, se internalizaron las reglas del juego (Juul, 2005) relacionadas 
con este tipo de construcciones que facilitan la solución de nuevos desafíos. Esto permite no sólo resolver los problemas con mayor facilidad, sino también enfrentarnos a problemas más complejos cuando éstos se plantean por primera vez, una vez que se identifica que lo que se debe hacer responde a un esquema ya conocido. No obstante, recurrir a un esquema previo o alcanzar un estado intermedio a veces no nos lleva a la solución, y es necesario dar un paso más para alcanzar la meta combinando ambas estrategias. En ese sentido y retomando la secuencia correspondiente a la construcción del tren, observamos que en este caso nos encontramos con una situación de ese tipo.

\section{Fragmento 5: Esquemas que se convierten en submetas}

200902 23. Sesión 2. Cámara móvil

(Continuación)

7. M: Que de curvas he hecho ¿no? Van a ir un poco... ¿Donde está? Ay, madre... ¿Qué es eso? ((hay un signo de rayo que titila)) (...)

8. P: No lo se ¡Profe! Cuando sale... ¿El rayito ese?

i. (...)

9. P: que cuando sale el rayito ese en la... en el tren ¿Qué pasa?

10. Prof.: Que no tiene electricidad. Tenéis que llevar también hacia el tren.

11. M: ¡Más! Madre mía...

12. P: Pues haz otro tendido de esos.

Como se observa en el diálogo, ha aparecido un "rayo" que indica que es necesario suministrar electricidad a la nueva construcción. Al desconocer su significado, los estudiantes solicitan ayuda al docente quien en el turno 10 señala que ese símbolo indica que el tren necesita electricidad. De esta forma, para alcanzar el estado final, la construcción del ferrocarril, además de lograr las dos submetas es necesario volver a resolver el problema de la electricidad. De esta forma el esquema ya creado para colocar la electricidad se constituye en una submeta de este problema. En este caso los alumnos han necesitado extender el tendido eléctrico y colocar una nueva central, ya que la que tenían no era suficiente para abastecer a la nueva red.

Por otra parte observamos que las reglas del juego son claras respecto a esto y se asemejan a una ciudad real, en donde es razonable pensar que al ampliar las infraestructuras se deberán también ampliar los servicios. La forma de señalar esto en el juego es a través de un rayo intermitente, que indica que es necesario suministrar energía a la nueva construcción. Conocer el significado de los símbolos que se presentan en el juego permite comprender la 
mecánica del mismo y en este caso, saber que remite directamente al esquema previamente internalizado del sistema eléctrico.

\section{Conclusiones}

Como señalamos al comenzar este artículo, pretendíamos analizar los procesos de resolución de problemas que se presentaban cuando se introduce en el aula un videojuego de simulación como Sim City Creator. Los datos presentados muestran cómo resolver los problemas que plantea el juego no es tarea sencilla. Construir una ciudad virtual requiere la puesta en marcha de procesos que impliquen crear una representación (Hayes, 1989) adecuada de los problemas que se plantan y llevar a cabo la secuencia de pasos tendientes a solucionarlos (Bassok \& Novick, 2012). Por una parte nos centramos en analizar el papel de los operadores y las condiciones (Newell \& Simon, 1972), mostrando como los mismos se presentaban en el videojuego y en el marco de nuestro estudio. Asimismo, estudiamos diferentes formas de resolverlos, ya sea mediante los métodos fraccionarios (Hayes, 1989) que permiten establecer submetas, o recurriendo a esquemas (Marshall, 1995) generados previamente.

A través de los resultados pudimos observar como en el caso de Sim City Creator, los operadores (Bassok \& Novick, 2012) se presentan en la pantalla, localizados en el menú del juego que se recorre mediante un mando inalámbrico. La presentación visual (Goh, Than \& Choy, 2012) de los operadores a través de dibujos presentes en el menú, hace que los estudiante cuenten con una referencia concreta a la que recurrir para resolver el problema. Cada icono del menú genera una acción diferente que puede hacer a los alumnos avanzar hacia la meta o por el contrario, elegir un camino equivocado. De allí la importancia de reconocer los operadores adecuados y saber que función realizará cada uno en el juego para elegir el correcto. Esta tarea no resultó sencilla para los participantes, quienes muchas veces encontraron dificultades para orientarse en los diferentes niveles del complejo menú del videojuego. Sin embargo, navegar por el espacio del problema en repetidas ocasiones y contar con la ayuda del docente, permitió adquirir la competencia necesaria para localizarlos.

Asimismo, resolver problemas utilizando videojuegos supone diferencias por ejemplo, frente a un problema matemático resuelto en lápiz y papel. En este caso, el espacio del problema cuenta con la interactividad propia de esta tecnología, aunque recorrerlo requiere de cierta destreza motora que como vimos, no es fácil alcanzar. Por momentos el propio juego no 
ayuda a encontrar el operador, sino que por el contrario distrae la atención de los jugadores/alumnos hacia el control del mando. No obstante, los fragmentos analizados reflejan que una vez que se conocen la finalidad y función de los diferentes operadores y se logra manejar el mando, la tarea es realizada, lo cual permitirá alcanzar la meta, pudiendo así resolver los problemas planteados por el juego.

En este proceso, el papel de las condiciones (Bassok \& Novick, 2012) también cobra relevancia. En nuestro estudio observamos como las mismas han sido planteadas tanto por los participantes, docente o alumnos, como por el mismo videojuego. Las primeras respondieron fundamentalmente a la experiencia previa y a la relación entre el mundo real y el mundo virtual, buscando similitudes con la vida real (Shaffer, 2006). El papel del docente como guía, acotando el espacio del problema con sus indicaciones, ha sido crucial para ayudar a los alumnos a construir representaciones correctas que permitieran resolver los diferentes problemas del juego. Por otra parte, hemos visto condiciones derivadas de las reglas del juego (Juul, 2005) que plantean lo que es posible o no dentro del mundo virtual del mismo. En relación con ello los fragmentos reflejaron, por ejemplo, que es necesario construir una central eléctrica y un tendido para que la ciudad tenga electricidad ya que sin ambos elementos no se logra colocar el servicio, que no se puede colocar un elemento sobre otro o que la aparición de un rayo indica que hace falta más electricidad en la zona. Es importante destacar que aunque el diseño del juego y sus metas sean abiertas, esto no impidió que los participantes tuvieran sus propias metas y aplicaran sus condiciones, que sumadas a las que se desprenden de las reglas del juego, permitieron representar los problemas y resolverlos.

Por otra parte se destaca la importancia de ciertas ayudas que colaboraron en la construcción de la representación del problema (Novick \& Bassok, 2005). En nuestro estudio éstas se hicieron presentes en algunos elementos del propio juego, como el mapa, que permitió a los alumnos orientarse en la ciudad virtual siendo una referencia constante al momento de tener que construir las infraestructuras y los servicios. Asimismo, observamos como la representación interna del docente, construida en base a su propia experiencia como jugador, funciona por una parte como representación externa para los alumnos, pero también como condición propia del problema, ya que acota el espacio del mismo, indicando una secuencia determinada de acciones a seguir (Hayes, 1989). 
De esta manera, observamos como las condiciones y los operadores adquieren cierta especificidad al encontrarse dentro del ámbito del videojuego, y asimismo, dentro del contexto áulico en el que se desarrolla la actividad. Construir una ciudad virtual también implicó trabajar con diferentes estrategias (Lacasa, 2011) para crear los elementos que la misma necesitaba. En ese sentido, observamos como el juego en su diseño plantea diferentes servicios e infraestructuras a crear que implican la construcción de varios elementos. De esta forma, los alumnos atravesaron el espacio del problema e identificaron cuáles eran esos elementos, estableciendo qué operadores emplear y qué condiciones seguir para lograr una correcta instalación de los mismos. De esta conjunción entre operadores y condiciones surgieron diferentes submetas (Hayes, 1989). Aproximarse de esta manera a los problemas les permitió aplicar los métodos de fraccionamiento (Hayes, 1989). Mediante éstos se analiza el problema tratando de encontrar cuáles pueden ser las submetas y cómo alcanzarlas. En el caso del juego observamos cómo los servicios en su mayoría requerían esta estrategia, por lo que ha sido empleada en diversas oportunidades.

Por otra parte, hemos observado la importancia de los esquemas (Marshall, 1995) para afrontar problemas nuevos. Al resolver los problemas del juego se internalizaron sus propiedades que luego estuvieron disponibles en forma de esquemas. De esta forma identificar puntos en común con otros problemas que se han resuelto en la partida, permitió recuperar esquemas creados previamente y utilizarlos para afrontar los nuevos (Novick \& Bassok, 2005). Recurrir a esta estrategia implica que se ha empleado el razonamiento por analogía (Gick \& Holyoak, 1980), al recuperar patrones similares e incluirlos en la representación del problema. De esta forma una estrategia exitosa que ha dado resultados positivos a la hora de resolver un problema, se internaliza y permanece disponible al enfrentarse a nuevos desafíos. Asimismo, hemos observado cómo en casos puntuales se han combinado las dos formas de acercarse al problema, la resolución de submetas y el uso de esquemas, siendo el esquema en si una submeta del problema.

A modo de conclusión final, los resultados expuestos son un ejemplo de cómo jugar con un videojuego no implica solamente un momento de ocio y diversión. El análisis de los datos nos ha permitido constatar que la construcción de los diferentes servicios e infraestructuras que requiere la ciudad virtual del videojuego Sim City Creator implica constantes procesos de resolución de problemas. Incluir un videojuego comercial dentro de un entorno educativo permitió al alumnado enfrentarse a la resolución de problemas diversos que tuvieron que 
representar y solucionar adecuadamente. Dada la importancia de la resolución de problemas en la vida diaria, consideramos que nuestro estudio demuestra el potencial de los videojuegos para desarrollar esos procesos y la relevancia de continuar profundizando en estas temáticas en futuros trabajos.

\section{Referencias}

Anderson-Levitt, K. M. (2006). Ethnography. In J. L. Green, G. Camilli \& P. B. Elmore (Eds.), Handbook of Complementary Methods in Education Research (pp. 279-296). Washington DC: AERA \& LEA.

Anfara, V., Brown, K., \& Mangione, T. (2002). Qualitative Analysis on Stage: Making the research Process More Public. Educational Researcher, 31, 28-38.

Baek, Y. (2009). Digital Simulation in Teaching and Learning. In D. Gibson \& Y. K. Baek (Eds.), Digital Simulations for Improving Education: Learning Through Artificial Teaching Environments (pp. 25-51). New York: Information Science Reference.

Bassok, M., \& Novick, L. (2012). Problem Solving. In K. J. Holyoak \& R. G. Morrison (Eds.), The Oxford Handbook of Thinking and Reasoning. New York: Oxford University Press.

Bechtel, W., \& Abrahamsen, A. (1991). Connectionism and the Mind: An Introduction to Parallel Processing in Networks. Cambridge: MA: Basil Blackwell.

Catrambone, R. (1996). Generalizing solution procedures learned from examples. Journal of Experimental Psychology: Learning, Memory and Cognition, 22.

de Vega, M. (1998). Introducción a la Psicología Cognitiva. Salamanca, España: Alianza Editorial.

Denzin, N. K., \& Lincoln, Y. S. (2011). Introduction: The discipline and practice of qualitative research. In N. K. Denzin \& Y. S. Lincoln (Eds.), The SAGE handbook of qualitative research (4 ed., pp. 1-21). Thousand Oaks, CA: Sage Publications.

Domin, D., \& Bodner, G. (2012). Using Students' Representations Constructed during Problem Solving To Infer Conceptual Understanding. Journal of Chemical Education, 89, 837-843.

Duncker, K. (1945). On Problem Solving. Psychological Monographs, 58 (American Psychological Association).

Egenfeldt Nielsen, S., Smith, J. H., \& Tosca, S. P. (2008). Understanding Video Games: The essential Introduction. New York: Routledge. 
Flyvbjerg, B. (2006). Five Misunderstandings About Case-Study Research. Qualitative Inquiry, 12, 219-244.

Gee, J. P. (2011). An Introduction to Discourse Analysis: Theory and Method ( $3^{\circ}$ Ed. ed.). New York, USA: Routledge.

Gee, J. P. (2007). What Video Games have to teach about learning and literacy. New York: Palgrave Macmillan.

Gee, J. P. (2008). Video games and Embodiment. Games and Culture, 3, 3-4.

Gee, J. P., \& Green, J. L. (1998). Discourse analysis, learning and social practice: A methodological study. Review of Research in Education, 23, 119-171.

Gick, M. L., \& Holyoak, K. J. (1980). Analogical Problem Solving. Cognitive Psychology, 12.

Goh, M., Tan, A.-G., \& Choy, W. (2012). The use of video source in analogical problem solving in two experimental studies. Procedia - Social and Behavioral Sciences, 31, 584588.

Hayes, J. (1989). The complete problem solver ( $2^{\circ}$ ed.). United States: Lawrence Erlbaum Inc. Hoepfl, M. (1997). Choosing Qualitative Research: A primer for Technology EducationResearch. Journal of Technology Education, 9(1).

Holyoak, K. J., \& Morrison, R. G. (2005). The Cambridge Handbook of Thinking and Reasoning. Cambridge: Cambridge University Press.

Holyoak, K. J. (2012). Analogy and Relational Reasoning. In K. J. Holyoak \& R. G. Morrison (Eds.), The Oxford Handbook of Thinking and Reasoning. New York: Oxford University Press.

Holyoak, K. J. (2005). Analogy. In K. J. Holyoak \& R. G. Morrison (Eds.), The Cambridge Handbook of Thinking and Reasoning. Cambridge: Cambridge University Press.

Johnson-Laird, P. N. (2008). Mental Models and Deductive Reasoning. In L. Rips \& J. Adler (Eds.), Reasoning: Studies in Human Inference and Its Foundantions. Cambridge: Cambidge University Press.

Jonassen, D. H. (2011). Learning to Solve Problems: A Handbook for Designing ProblemSolving Learning Environments. New York: Routledge.

Juul, J. (2005). Video games and the classic game model Half-Real: Video games between Real rules and Fictional worlds. Cambidge, UK: MIT press. 
Lacasa, P., \& GIPI. (2009). Videojuegos en el Instituto. Ocio digital como estímulo en la enseñanza. Madrid: Universidad de Alcalá, Universidad Nacional de Educación a Distancia.

Lacasa, P., Méndez, L. \& Martínez, R., (2008). Developing new literacies using commercial video games as educational tools. Linguistics and Education, 19(2).

Lacasa, P., \& y Grupo Imágenes, Palabras e Ideas (2006). Aprendiendo periodismo digital: historias de pequeñas escritoras. Madrid: Machado Libros.

Lacasa, P., \& Reina, A. (2004). La televisión y el periódico en la escuela primaria: Imágenes, palabras e ideas. Madrid: Ministerio de Educación, Cultura y Deporte. Centro de Investigación y Documentación Educativa.

Lee, C. B., Koh, N. K., Cai, X. L., \& Quek, C. L. (2012). Children's use of metacognition in solving everyday problems: Childrens monetary decision-making. Australia Journal of Education, 56(1), 23-40.

LeCompte, M., \& Goetz, J. (1982). Problems of Reliability and Validity in Ethnographic Research. Review of educational research, 52, 31-60.

Marshall, S. P. (1995). Schemas in problem solving. Australia: Cambridge University Press.

Monjelat, N. (2009). Construyendo ciudades virtuales. Retrieved from http://www.aprendeyjuegaconea.com/index.php?n3=42

Newell, A., \& Simon, H. A. (1972). Human problem solving. Englewood Cliffs, NJ: PrenticeHall.

Novick, L. R., \& Bassok, M. (2005). Problem Solving. In K. J. Holyoak \& R. G. Morrison (Eds.), The Cambridge Handbook of Thinking and Reasoning. Cambridge: Cambridge University Press.

Pink, S. (2007). Doing Visual Ethnography: Images, Media and Representation in Research. London: SAGE.

Pizlo, Z., \& Goldstone, R. (2009). New perspectives on Human Problem Solving. The Journal of Problem Solving, 2(1).

Potter, H. (2000). Post-cognitive Psychology. Theory \& Psychology, 10(1).

Prensky, M. (2001). Digital Game-Based Learning. London: McGraw-Hill.

Prensky, M. (2002). The motivation of gameplay: the real twenty-first century learning revolution. On the Horizons, 10(1), 5-11.

Ragin, C., \& Becker, H. (1992). What is a case? Exploring the foundantions os social inquiry. Cambridge, UK: Cambridge University Press.

Robertson, S. I. (2001). Problem Solving. Philadelphia: Psychology Press. 
Rumelhart, D. E. (1989). The Architecture of Mind: A Connectionist Approach. In M. Posner (Ed.), Foundations of Cognitive Science. Cambridge: MIT Press.

Salen, K., \& Zimmerman, E. (2004). Rules os play: Game Design Fundamentals. Cambridge, Massachusets

Sandford, R., \& Williamson, B. (2005). Games and learning. Bristol, United Kingdom.

Shaffer, D. W. (2006). How computer games help children learn. New York: Palmgrave Macmillan.

Simon, H. A. (1978). Informaction-Processing Theory oh Human Poblem Solving. In W. K. Estes (Ed.), Handbook of learning and cognitive processes (Vol. 5, pp. 271-295). NJ: Hillsdale.

Spindler, G., \& Hammond, L. (2006). Innovations in educational ethnography: Theory, methods and results. New York: LEA.

Squire, K. (2003). Video games in education. International Journal of Intelligent Simulations and Gaming, 2 (1).

Stake, R. (2006). Multiple case study analysis. New York: Guilford Press.

Stake, R. (1995). The Art of Case Study Research. Thousands Oaks, CA: Sage.

Sun, R. (2008). Introduction to Computational Cognitive Modeling. In R. Sun (Ed.), The Cambridge Handbook of Computactional Psychology. NY: Cambdridge University Press.

Thomas, M., \& McClelland, J. (2008). Connectionist Models of Cognition. In R. Sun (Ed.), The Cambridge Handbook of Compitational Psychology. NY: Cambridge University Press.

van de Laar, T. \& Regt, H. (2008). Special Section: Is Cognitive Science Changing its Mind?: Introduction to Embodied Embedded Cognition and Neurophenomenology. Theory \& Psychology, 18(3).

Vega, M. d. (1998). Introducción a la Psicología Cognitiva. Salamanca, España: Alianza Editorial.

Willatts, P. (1990). Development of prohlem-solving strategies in infancy. In D. F. Bjorklund (Ed.), Children's strategies. Contemporary views of cognitive development. Hillsdale, NJ: Larence Erlhaum Associates.

Yin, R. K. (2011). Applications of case study research (3. ed. ed.). Thousand Oaks: SAGE. 
Natalia Monjelat et al.

[Página en blanco por razones de paginación] 